\title{
ARTICLE
}

Clinical Study

\section{Phase III randomised trial comparing 6 vs. 12-month of capecitabine as adjuvant chemotherapy for patients with stage III colon cancer: final results of the JFMC37-0801 study}

Naohiro Tomita ${ }^{1}$, Katsuyuki Kunieda ${ }^{2}$, Atsuyuki Maeda ${ }^{3}$, Chikuma Hamada $^{4}$, Takeharu Yamanaka ${ }^{5}$, Toshihiko Sato ${ }^{6}$, Kazuhiro Yoshida $^{7}$ Narikazu Boku ${ }^{8}$, Riichiro Nezu ${ }^{9}$, Shigeki Yamaguchi ${ }^{10}$, Hideyuki Mishima ${ }^{11}$, Sotaro Sadahiro ${ }^{12}$, Kei Muro ${ }^{13}$, Megumi Ishiguro ${ }^{14}$, Junichi Sakamoto $^{15}$, Shigetoyo Saji ${ }^{16}$ and Yoshihiko Maehara ${ }^{17}$

BACKGROUND: Up to 6-months oxaliplatin-containing regimen is now widely accepted as a standard adjuvant chemotherapy for stage III colorectal cancer (CRC). However, oral fluoropyrimidine monotherapy is used for some part of patients, especially in Asian countries including Japan, and its optimal duration is yet to be fully investigated.

METHODS: A total of 1306 patients with curatively-resected stage III CRC were randomly assigned to receive capecitabine (2500 $\mathrm{mg} / \mathrm{m}^{2} /$ day) for 14 out of 21 days for $6(n=654)$ or $12(n=650)$ months. The primary endpoint was disease-free survival (DFS), and the secondary endpoints were relapse-free survival (RFS), overall survival (OS), and adverse events.

RESULTS: The 3- and 5-year DFS were $70.0 \%$ and $65.3 \%$ in the $6 \mathrm{M}$ group and $75.3 \%$ and $68.7 \%$ in the $12 \mathrm{M}$ group, respectively ( $p=$ $0.0549, \mathrm{HR}=0.858,90 \% \mathrm{Cl}: 0.732-1.004)$. The 5 -year RFS was $69.3 \%$ and $74.1 \%$ in the $6 \mathrm{M}$ and $12 \mathrm{M}$ groups, respectively $(p=0.0143$, $\mathrm{HR}=0.796,90 \% \mathrm{Cl}: 0.670-0.945)$. The 5 -year OS was $83.2 \%$ and $87.6 \%$, respectively $(p=0.0124, \mathrm{HR}=0.727,90 \% \mathrm{Cl}: 0.575-0.919)$. The incidence of overall grade 3-4 adverse events was almost comparable in both groups.

CONCLUSIONS: Although 12-month adjuvant capecitabine did not demonstrate superior DFS to that of 6-month, the observed better RFS and OS in the 12-month treatment period could be of value in selected cases.

British Journal of Cancer (2019) 120:689-696; https://doi.org/10.1038/s41416-019-0410-0

\section{BACKGROUND}

Colorectal cancer (CRC) is among the most common malignancies worldwide. Annually, >1,350,000 new cases are diagnosed, and approximately 700,000 people die from this disease. ${ }^{1}$ In Japan, the incidence of CRC cases has increased recently, with approximately 149,500 new CRC cases reported in $2017 .^{2}$

Surgery is the main treatment for CRC, while postoperative adjuvant chemotherapy is used to reduce recurrence and improve prognosis. Three previous studies-the NCCTG $894651,{ }^{3}$ INT-0089 ${ }^{4}$ and another ${ }^{5}$ studies-evaluated drug selection and administration for postoperative adjuvant chemotherapy for CRC. All these studies showed no significant differences in disease-free survival (DFS) between 6 month and longer duration of 5-FU-based regimens. In the $\mathrm{X}-\mathrm{ACT} \mathrm{T}^{6}$ and $\mathrm{NSABPC}-06^{7}$ studies, capecitabine and UFT-LV, respectively, were compared with 5-FU/LV for 6 months. Based on these studies, 6 months was determined to be the standard treatment period for postoperative adjuvant chemotherapy for CRC.

However, given the lack of sufficient detection sensitivity during the treatment periods and the differences in the treatment schedules among these studies, the National Cancer Institute Physician Data Query stated that the current evidence supporting this treatment period is not definitive. ${ }^{8}$ Moreover, a meta-analysis indicated that 1-year administration of oral 5-FU in stage III CRC achieved significantly superior DFS and overall survival (OS) to surgery alone, ${ }^{9}$ and the period of administration for oral 5-FU was set as $\geq 1$ year in numerous Japanese clinical trials. Thus, whether 6 months is the optimal treatment period for oral 5-FU drugs as the adjuvant postoperative chemotherapy for CRC remains inconclusive.

By analysing the year-to-year hazard rate of recurrence after curative surgery, Hamada et al. ${ }^{10}$ recently suggested that

\footnotetext{
${ }^{1}$ Department of Surgery, Division of Lower Gastrointestinal Surgery, Hyogo College of Medicine, Hyogo, Japan; ${ }^{2}$ Department of Surgery, Gifu Prefectural General Medical Center, Gifu, Japan; ${ }^{3}$ Department of Surgery, Ogaki Municipal Hospital, Gifu, Japan; ${ }^{4}$ Graduate School of Engineering, Tokyo University of Science, Tokyo, Japan; ${ }^{5}$ Department of Biostatistics, Yokohama City University School of Medicine, Kanagawa, Japan; ${ }^{6}$ Yamagata Prefectural Central Hospital, Yamagata, Japan; ${ }^{7}$ Department of Surgical Oncology, Gifu University, Graduate School of Medicine, Gifu, Japan; ${ }^{8}$ National Cancer Center Hospital, Tokyo, Japan; ${ }^{9}$ Nishinomiya Municipal Central Hospital, Hyogo, Japan; ${ }^{10}$ Department of

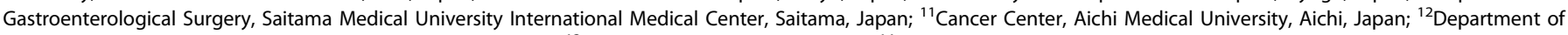
Surgery, Tokai University School of Medicine, Kanagawa, Japan; ${ }^{13}$ Aichi Cancer Center, Aichi, Japan; ${ }^{14}$ Department of Translational Oncology, Tokyo Medical and Dental University

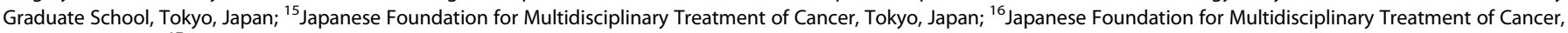
Tokyo, Japan and ${ }^{17}$ Japanese Foundation for Multidisciplinary Treatment of Cancer, Tokyo, Japan
}

Correspondence: Naohiro Tomita (ntomita@hyo-med.ac.jp)

Chikuma Hamada is deceased.

Received: 6 August 2018 Revised: 11 January 2019 Accepted: 4 February 2019

Published online: 5 March 2019 
prolonged administration of oral 5-FU might improve the prognosis of patients with CRC. The recurrence risk in the three study groups peaked between 1 and 2 years postoperatively. However, no peak recurrence risk was observed in the 1-year oral 5-FU drug therapy (drugs used: UFT and HCFU) group.

Although 6-month adjuvant chemotherapy is the current standard treatment modality for patients with stage III CRC, whether prolonged chemotherapy, particularly using oral fluoropyrimidine, can improve patient survival remains unclear.

Aside from fluoropyrimidines, the new agent oxaliplatin has become one of the primary adjuvant chemotherapeutic for CRC. Based on the results of three randomised controlled trialsMOSAIC, $^{11}$ NSABP C-07, ${ }^{12}$ and XELOXA ${ }^{13}$-infusional fluorouracil and folinic acid with oxaliplatin (FOLFOX) and capecitabine with oxaliplatin (CapeOX) have become the standard postoperative adjuvant chemotherapy for $\mathrm{CRC}^{14}$ Six-month oxaliplatin-based treatment might be an ideal control arm to investigate the potential role of prolonged administration of 5-FU in randomised controlled studies. However, in Japan, oxaliplatin could not be used when the current trial was designed because it only became available in August 2009. Therefore, we adopted 6-month capecitabine as the standard treatment for investigating the clinical utility of prolonged oral 5-FU administration in the adjuvant setting.

Herein, we report the final results of our multi-institutional randomised controlled trial comparing 6 vs. 12 months of capecitabine as adjuvant chemotherapy for patients with stage III CRC (JFMC37-0801).

The safety and feasibility of the 12-month capecitabine regimen in this study was previously reported. ${ }^{15}$

\section{PATIENTS AND METHODS}

\section{Study design}

JFMC37-0801 was a multi-institutional, open-label, randomised, phase III study (see Supplementary Figure 1). It was conducted in accordance with the Declaration of Helsinki and the Ethical Guidelines for Clinical Research in Japan and was approved by the Institutional Review Boards of each participating institute. Written informed consent was obtained from all patients before their enrolment, and then the eligible patients were centrally registered. This study primarily aimed to demonstrate the superiority of adjuvant 12 months of capecitabine (16 courses) to 6 months (eight courses) in terms of DFS for stage III (Dukes' C) CRC after curative resection. Secondary endpoints were relapse-free survival (RFS), OS, and safety.

A complete study protocol of JFMC-37 trial is shown in Supplementary file.

\section{Enrolment and assignment}

The main eligibility criteria were as follows: (1) histologically confirmed stage III colon adenocarcinoma; (2) curatively resected with extended lymph node dissection (D2 or D3 in the Japanese Classification of Colorectal Carcinoma, 7th edition); ${ }^{15}$ (3) aged 20-79 years; (4) Eastern Cooperative Oncology Group performance status (ECOG-PS) of $0-1 ;(5)$ no prior chemotherapy or radiotherapy for $\mathrm{CRC}$; (6) no other active malignancies; (7) adequate oral intake; (8) preserved major organ functions and, (9) no uncontrollable severe infection.

\section{Randomisation and masking}

After confirming eligibility, enrolled patients were randomly assigned to receive either 6 (eight cycles) or 12 (16 cycles) months of capecitabine using a minimisation method, with stratification according to lymph node metastasis (N1 or N2-3 in the Japanese Classification of Colorectal Carcinoma, 7th edition) ${ }^{16}$ and institution. The assigned treatment arm was not blinded from both investigators and patients.
Protocol treatment

Oral capecitabine was administered at a dose of $1250 \mathrm{mg} / \mathrm{m}^{2}$ twice daily after meals for 14 consecutive days, followed by a 7 day rest. This 3-week treatment comprised one course. The control group (6M) and study group (12M) received 8 and 16 courses, respectively. The assigned treatment was started within 8 weeks after surgery. During treatment, clinical findings and laboratory values were evaluated at least every 3 weeks. Evaluation at the beginning of each cycle was mandatory. Patients received treatment if they fulfilled the following criteria: leucocytes $\geq 3000 / \mathrm{mm}^{3}$, neutrophils $\geq 1500 / \mathrm{mm}^{3}$, platelets $\geq 75,000 / \mathrm{mm}^{3}$, aspartate aminotransferase and alanine aminotransferase $\leq 2.5 \times$ upper limit of normal (ULN), total bilirubin $\leq 1.5$ ULN, creatinine $<$ $1.5 \times \mathrm{ULN}$, and no $>$ grade 1 non-haematologic toxicities (i.e., anorexia, nausea, vomiting, and diarrhoea). If the criteria for starting/continuing treatment course were not fulfilled, treatment was postponed or temporarily suspended until adverse events (AEs) improved sufficiently to meet the criteria.

Depending on the severity of AEs, the dose of capecitabine was reduced according to the protocol. When a grade $2 \mathrm{AE}$ developed for the first time, treatment with capecitabine was suspended until the $A E$ improved to grade $\leq 1$, and then resumed at the same dose. If a grade $2 \mathrm{AE}$ occurred twice or more and if a grade $3 \mathrm{AE}$ occurred, the dose of capecitabine was reduced by $25 \%$. The minimum dose of $50 \%$ of the initial dose was allowed in the protocol.

The treatment was discontinued in the following: (1) consent was withdrawn; (2) treatment was requested to be discontinued; (3) recurrence of the original disease; (4) newly detected cancerous lesions (duplicated cancer or multifocal CRC [excluding T1a cancer]); (5) as judged by the attending physician due to deterioration of $A E$, comorbidities, onset of complications, or other reasons; (6) $\mathrm{AE}$ requiring dose delay (>3 weeks) beyond allowance pre-specified in the protocol; (7) $A E$ requiring dose reduction > 50\%; (8) Grade $4 \mathrm{AE}$; (9) death; (10) transfer to other hospital; (11) ineligibility after enrolment. If capecitabine treatment is restarted after protocol termination, documentation must be submitted.

Primary/secondary endpoint analyses and statistical basis The primary endpoint was DFS, which is defined as survival from the randomisation without the following events; (1) occurrence of secondary cancer (including CRCs and any other malignancies), (2) recurrence, and (3) death due to any cause. The secondary endpoints included RFS, OS, and safety. RFS was defined as the period without (2) and (3), and OS was without (3). Patients with no events at the final data cut-off were censored.

The DFS, RFS, and OS were estimated by group using the Kaplan-Meier method. Greenwood's formula was used for interval estimation. The stratified log-rank test was used to evaluate the hypothesis. The stratified Cox proportional hazard model will be used to calculate hazard ratio. The hazard ratio of the treatment effect among the groups and its $90 \%$ confidence interval will also be obtained.

A Wald-type estimator was used to estimate confidence intervals. An adjusted hazard ratio accounting for other background factors would be derived as necessary. A similar secondary analysis will be performed for all eligible or treated subjects. As for secondary endpoint analyses, RFS and OS were evaluated using the same analysis as DFS. Chi-square test was used to compare the incidence of AEs between the treatment groups.

A one-tailed $p<0.0477$ was considered significant in the primary endpoint analysis. Because we conducted the interim analysis at information time 0.53125 (255/480 events), 0.0022 of alpha error was spent. Therefore, if the one-sided significance level would be $<0.0478$, the $12 \mathrm{M}$ duration would be the standard 


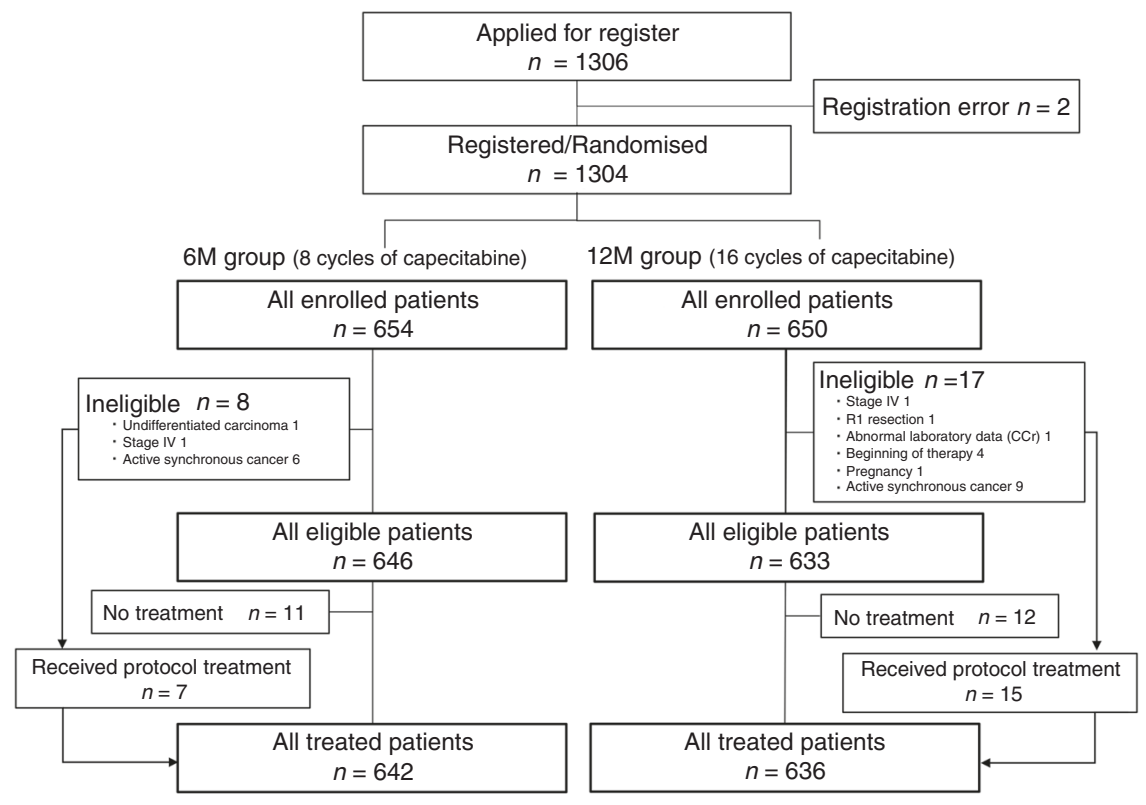

Fig. 1 CONSORT diagram

Table 1. Baseline patient characteristics ( $N=1304$ intension-to-treat population)

\begin{tabular}{|c|c|c|c|}
\hline \multicolumn{2}{|l|}{ Total number } & \multirow{2}{*}{$\begin{array}{l}\begin{array}{l}6 \mathrm{M} \text { group } \\
n=654\end{array} \\
504(77.1 \%)\end{array}$} & \multirow{2}{*}{$\begin{array}{l}\begin{array}{l}12 \mathrm{M} \text { group } \\
n=650\end{array} \\
498(76.6 \%)\end{array}$} \\
\hline Nodal status & N1 & & \\
\hline & N2/N3 & $150(22.9 \%)$ & $152(23.4 \%)$ \\
\hline \multirow[t]{2}{*}{ Gender } & Male & $352(53.8 \%)$ & $343(52.8 \%)$ \\
\hline & Female & $302(46.2 \%)$ & $307(47.2 \%)$ \\
\hline \multirow[t]{2}{*}{ Age } & $<70$ & $451(69.0 \%)$ & $442(68.0 \%)$ \\
\hline & $\geqq 70$ & $203(31.0 \%)$ & $208(32.0 \%)$ \\
\hline \multirow[t]{3}{*}{ Tumour location } & Right-sided colon $(\mathrm{C} / \mathrm{A} / \mathrm{T})$ & $262(40.1 \%)$ & $263(40.5 \%)$ \\
\hline & Left-sided colon (D/S) & $258(39.4 \%)$ & $252(38.8 \%)$ \\
\hline & Rectosigmoid colon & $134(20.5 \%)$ & $135(20.8 \%)$ \\
\hline \multirow{2}{*}{$\begin{array}{l}\text { Surgical } \\
\text { approach }\end{array}$} & Laparoscopic & $276(42.2 \%)$ & 255 (39.2\%) \\
\hline & Open(conventional) & $378(57.8 \%)$ & $395(60.8 \%)$ \\
\hline \multirow{3}{*}{$\begin{array}{l}\text { Histological } \\
\text { type }\end{array}$} & Pap/ Well & 189 (28.9\%) & $178(27.4 \%)$ \\
\hline & Tub/Mod & 419 (64.1\%) & $433(66.6 \%)$ \\
\hline & $\begin{array}{l}\text { Poor/Solid/Mon-solid/ } \\
\text { Muc/Sig/Other }\end{array}$ & $46(7.0 \%)$ & $39(6.0 \%)$ \\
\hline \multirow[t]{3}{*}{$\mathrm{T}\left(\mathrm{TMN} 7^{\mathrm{th}}\right)$} & $\mathrm{T} 1 / \mathrm{T} 2$ & $101(15.4 \%)$ & $100(15.4 \%)$ \\
\hline & T3 & $366(56.0 \%)$ & $363(55.8 \%)$ \\
\hline & T4 & $187(28.6 \%)$ & $187(28.8 \%)$ \\
\hline \multirow[t]{2}{*}{$N\left(\right.$ TNM $\left.7^{\text {th }}\right)$} & $\mathrm{N} 1$ & $512(78.3 \%)$ & $506(77.8 \%)$ \\
\hline & N2 & $142(21.7 \%)$ & $144(22.2 \%)$ \\
\hline \multirow[t]{3}{*}{ Stage (TNM $7^{\text {th }}$ ) } & IIIA & $94(14.4 \%)$ & $96(14.8 \%)$ \\
\hline & IIIB & 461 (70.5\%) & $458(70.5 \%)$ \\
\hline & IIIC/IVA/IVB & 99 (15.1\%) & 96 (14.8\%) \\
\hline
\end{tabular}

therapy. In other tests, $p<0.05$ was considered significant. All statistical analyses were performed using SAS software version 9.3 (SAS Institute, Cary, NC, USA). Descriptive statistics were calculated.
Subjects and statistical basis

There should be no difference in DFS for first 6-month period because the $6 \mathrm{M}$ and $12 \mathrm{M}$ groups received the same treatment after randomisation, with differences expected to appear 6 months or later. When calculating the number of subjects assuming a normal exponential distribution without considering the above, the number of required subjects is underpowered. Therefore, in this study, the number of subjects was set assuming a segmented exponential model.

In the 6 months following randomisation, the hazard ratio for DFS groups would be 1.0 and assuming that differences in DFS would begin to appear after 6 months or later. Presupposing that the 5-year DFS rate in the $6 \mathrm{M}$ and $12 \mathrm{M}$ groups would be $60 \%$ and $67 \%$, respectively, 1142 subjects (480 events) were required, with a one-sided significance level of $5 \%$ and a detection power of $80 \%$. Finally, the number of target subjects are determined to be 1200 (600 patients per group), assuming that $\sim 5 \%$ will drop out.

\section{RESULTS}

Patient characteristics

A total of 1306 patients were enrolled from 333 institutions in Japan between September 2008 and December 2009. Excluding two patients due to registration error, 1304 patients were randomly assigned to the two groups: 654 patients in the $6 \mathrm{M}$ group (control arm) and 650 patients in the 12M group (study arm). Eight and 17 patients were ineligible and 11 and 12 did not receive the protocol treatment in the $6 \mathrm{M}$ and $12 \mathrm{M}$ groups, respectively. The reasons for ineligibility are provided in Fig. 1.

Based on a scheduled intention-to-treat analysis, all enrolled patients including those who were ineligible were included in the final efficacy analysis set. Patient demographics were well balanced in the two groups (Table 1). A total of 1278 patients (642: 6M group; 636: 12M group) who finally received capecitabine were included in the safety analysis set. All data for the safety analyses were finalised in March 2016.

Disease-free survival

DFS was analysed based on 60.6 months of median follow-up with 434 events ( 226 and 208 events in the $6 \mathrm{M}$ and $12 \mathrm{M}$ groups, 
a

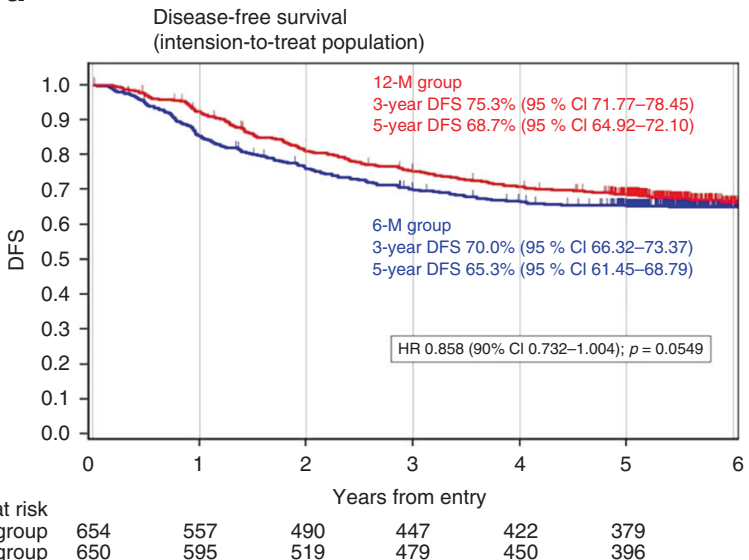

b

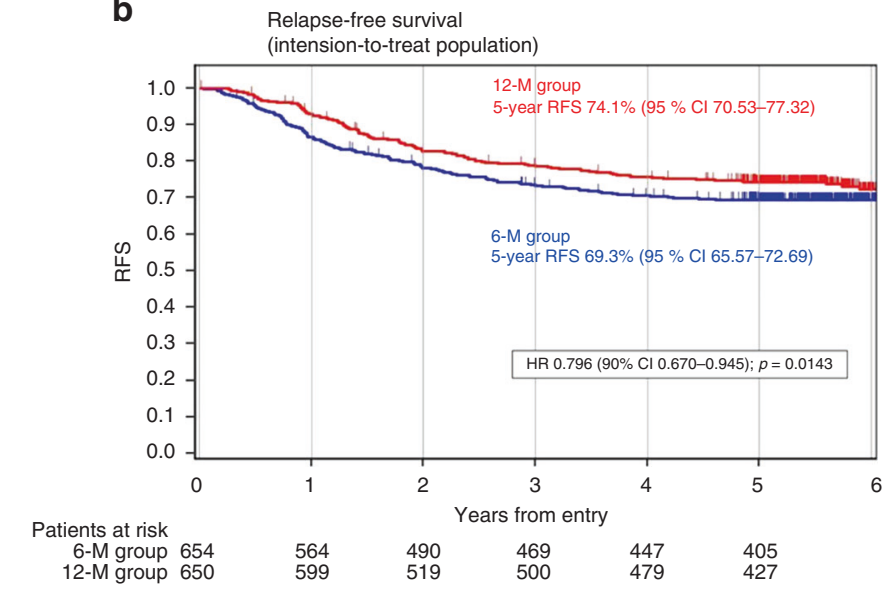

C Overall survival

(intension-to-treat population)

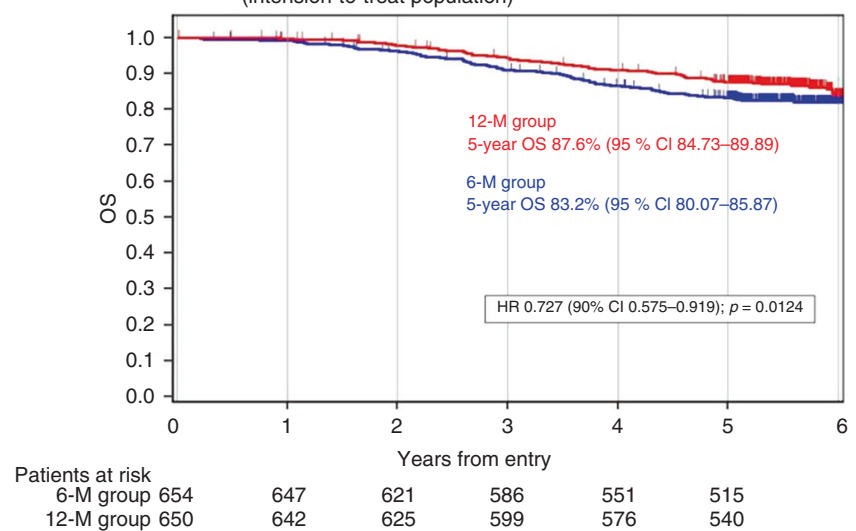

Fig. 2 a Disease-free survival rate in all enrolled patients. b Relapse-free survival rate in all enrolled patients. c Overall survival rate in all enrolled patients

respectively). The 5-year DFS rate was $65.3 \%$ (95\% Cl: 61.45-68.79) and $68.7 \%$ (95\% Cl: $64.92-72.10)$ in the $6 \mathrm{M}$ and $12 \mathrm{M}$ groups, respectively. The HR was $0.858(90 \% \mathrm{Cl}: 0.732-1.004 ; p=0.0549)$. No statistical difference in DFS was noted between the $12 \mathrm{M}$ and $6 \mathrm{M}$ group with the significance level of 0.0478 (Fig. 2a, Table 2). HR in all eligible and treated patients was $0.833(90 \%$ Cl: $0.710-0.979 ; p=0.0308)$ and 0.848 (90\% Cl: 0.722-0.995; $p=$ $0.0452)$, respectively.

DFS Kaplan-Meier curve according to substage of stage III CRC was also shown in Supplementary Figure 2.

\section{Relapse-free survival}

RFS was analysed based on 61.0 months of median follow-up with 368 events (199 and 169 events in the $6 \mathrm{M}$ and $12 \mathrm{M}$ groups, respectively). The 5-year RFS rate was $69.3 \%$ (95\% Cl: 65.57-72.69) and $74.1 \%$ (95\% Cl: $70.53-77.32)$ in the $6 \mathrm{M}$ and $12 \mathrm{M}$ groups, respectively. The HR was 0.796 (90\% Cl: $0.670-0.945 ; p=0.0143$ ). RFS in the $12 \mathrm{M}$ group was statistically superior to that of the $6 \mathrm{M}$ group (Fig. 2b, Table 2).

Recurrence was reported in 339 (26\%) patients (6M group: 184 [28.1\%]; $12 \mathrm{M}$ group: 155 [23.8\%]). The most common site of recurrence in the $6 \mathrm{M}$ and $12 \mathrm{M}$ groups was the liver (76 (11.6\%) and 59 (9.1\%), respectively).

Hazard rate of recurrence

The biweight kernel smoothing hazard function of RFS in the $6 \mathrm{M}$ and $12 \mathrm{M}$ groups is shown in Fig. 3 . The hazard rate peaked at $\sim 0.8$ year and 1.2 year after registration in the $6 \mathrm{M}$ and $12 \mathrm{M}$ groups, respectively. Within 1.5 years, the hazard rate in the $6 \mathrm{M}$ group was higher than that of the $12 \mathrm{M}$ group; thereafter, they were similar. As regards DFS in the $6 \mathrm{M}$ group, the risk of events was evidently the highest during the first year after surgery, followed by a rapid reduction until approximately 5 years. The risk in the $12 \mathrm{M}$ group in 1 year was lower than that in the $6 \mathrm{M}$ group. The difference in the hazard rate between the two groups from year 0.5 to 1.5 was statistically significant $(\mathrm{HR}=0.713 ; p=0.0149)$.

Overall survival

OS was analysed based on 63.3 months of median follow-up with 200 events (113 and 87 in the $6 \mathrm{M}$ group and $12 \mathrm{M}$ group, respectively). The 5 -year OS was $83.2 \%$ (95\% Cl: $80.07-85.87)$ and $87.6 \%(95 \% \mathrm{Cl}: 84.73-89.89)$ in the $6 \mathrm{M}$ and $12 \mathrm{M}$ groups, respectively. The HR was 0.727 ( $90 \% \mathrm{Cl}: 0.575-0.919 ; p=0.0124)$. The OS in the $12 \mathrm{M}$ group was statistically superior to that in the $6 \mathrm{M}$ group (Fig. 2c, Table 2).

Subgroup analysis

Unstratified subgroup analyses of DFS were performed for nodal status (N1, N2/N3), sex (male, female), age $(<70, \geq 70)$, tumour location (right-sided colon, left-sided colon, rectosigmoid colon), surgical approach (laparoscopic, open), histological type (papillary/well differentiated, tubular/moderately differentiated, poorly differentiated/mucinous/signet ring cell/other), tumour depth (T1/T2, T3, T4, according to the 7th TNM classification), lymph 


\begin{tabular}{|c|c|c|c|c|c|c|}
\hline \multirow{2}{*}{ DFS } & $6 \mathrm{M}$ group & 654 & 226 & - & - & \\
\hline & $12 \mathrm{M}$ group & 650 & 208 & 0.858 & $0.732-1.004$ & \\
\hline RFS & $12 \mathrm{M}$ group & 650 & 169 & 0.796 & $0.670-0.945$ & \\
\hline \multirow[t]{3}{*}{ OS } & & & & & & $p=0.0124$ \\
\hline & $6 \mathrm{M}$ group & 654 & 113 & - & - & \\
\hline & $12 \mathrm{M}$ group & 650 & 87 & 0.727 & $0.575-0.919$ & \\
\hline
\end{tabular}

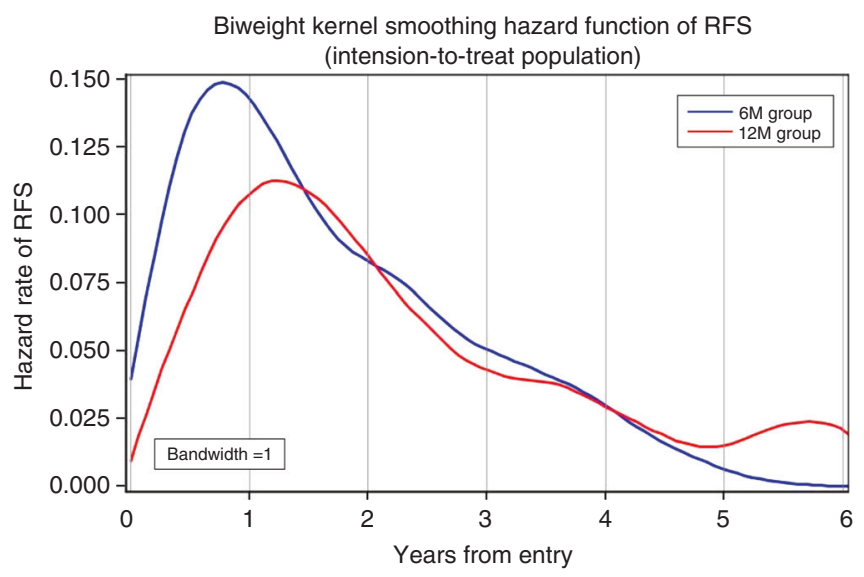

Fig. 3 Biweight kernel smoothing hazard function. Relapse-free survival was exploratorily analysed as hazard of recurrence

node metastasis (N1, N2, according to the 7th TNM classification), and TNM stage (IIIA, IIIB, IIIC/IVA/IVB, according to the 7th edition) (Fig. 4). The result showed a reduced risk of recurrence in the $12 \mathrm{M}$ group compared to the $6 \mathrm{M}$ group, particularly in the male sex, open surgical approach, and the T4 subgroup.

Chemotherapy and safety

The completion rate which was defined as the ratio of patients who completed 6 or 12 months of capecitabine to the number of patients included in the safety analysis set of each treatment group was $71.5 \%$ and $46.1 \%$ in the $6 \mathrm{M}$ and $12 \mathrm{M}$ groups, respectively. Details of the safety analysis have been reported previously. ${ }^{15}$ The average total dose of capecitabine for $6 \mathrm{M}$ group was $1169 \pm 409$ tablets (mean \pm SD) and that for $12 \mathrm{M}$ group was $1786 \pm 910$ tablets (mean \pm SD). One tablet corresponds to $300 \mathrm{mg}$ of capecitabine.

Briefly, the overall incidence rate of AEs was $91.7 \%$ and $94.7 \%$ in the $6 \mathrm{M}$ and $12 \mathrm{M}$ groups, respectively. The most common $\mathrm{AE}$ was hand-foot syndrome (HFS). Twelve months of adjuvant capecitabine demonstrated a higher cumulative incidence of HFS than the standard 6-month treatment; meanwhile, while toxicities even after 12-month capecitabine were clinically acceptable.

\section{DISCUSSION}

Recently, the final result of the JFMC33-0502 study was reported. ${ }^{17}$ It failed to show the superiority of 18 months adjuvant chemotherapy over 6 months regimen; however, because both stage IIB and III patients were included, and different drug intensities were used due to different administration schedule between the two arms in this study, and the results should be interpreted cautiously.

To the best of our knowledge, the present study is the first phase III trial on postoperative adjuvant chemotherapy in which the duration of oral 5-FU drug for CRC was directly compared. Primarily, 12 months capecitabine did not show superiority to 6 months regimen in terms of DFS. However, 12 months capecitabine showed significant superiority to 6 months both in RFS and OS. DFS events were defined as newly diagnosed cancer curable via local therapy. Our results show that adjuvant chemotherapy with capecitabine for 12 months has substantial clinical impact.

There are several possible explanations for the unmet primary result of this study. First, in the process of sample size estimation, we have assumed that 5-year DFS of $6 \mathrm{M}$ capecitabine would be $60 \%$ based on the result of the X-ACT study ${ }^{5}$ and that of $12 \mathrm{M}$ capecitabine would be $67 \%$, which was based on our expectation without any solid evidence. Actually, the 5-year DFS rate was substantially higher than we assumed (6M: $65.3 \%$ vs $12 \mathrm{M}: 68.7 \%)$.

Second, the survival curves of two groups are not diverging over time but gradually getting closer. One possible explanation is that the longer administration of capecitabine might be effective especially in cases with a high risk of recurrence and just delay recurrence rather than cure.

Third, because we could have detected statistical difference in DFS when analysed both in all eligible cases and in all treated patients, these excluded ineligible and/or not-treated cases should have negative impacts on the difference in efficacy between $6 \mathrm{M}$ and $12 \mathrm{M}$ capecitabine.

Fourth, the statistical method used in this study might be inappropriate to analyse DFS. As shown in Fig. 2a, considering that the DFS curves became separated initially then later became closer, the log-rank test used in this study might not be necessarily suitable. Although not pre-planned, we also analysed DFS using stratified generalised Wilcoxon test and obtained statistically significant results $(p=0.0343$ in all enrolled patients, $p=0.0151$ in all eligible patients, and $p=0.0232$ in all treated patients).

One limitation of this study was not adopting the 6 months oxaliplatin-based regimen, which is the current gold standard for adjuvant treatment of stage III CRC. This trial seems to deal with 2 non-standard therapies. However, oxaliplatin was not available as adjuvant treatment for CRC in Japan when this trial was planned. In addition, oral fluoropyrimidines alone has been used as 
Subgroup analysis of disease-free survival (intension-to-treat population)

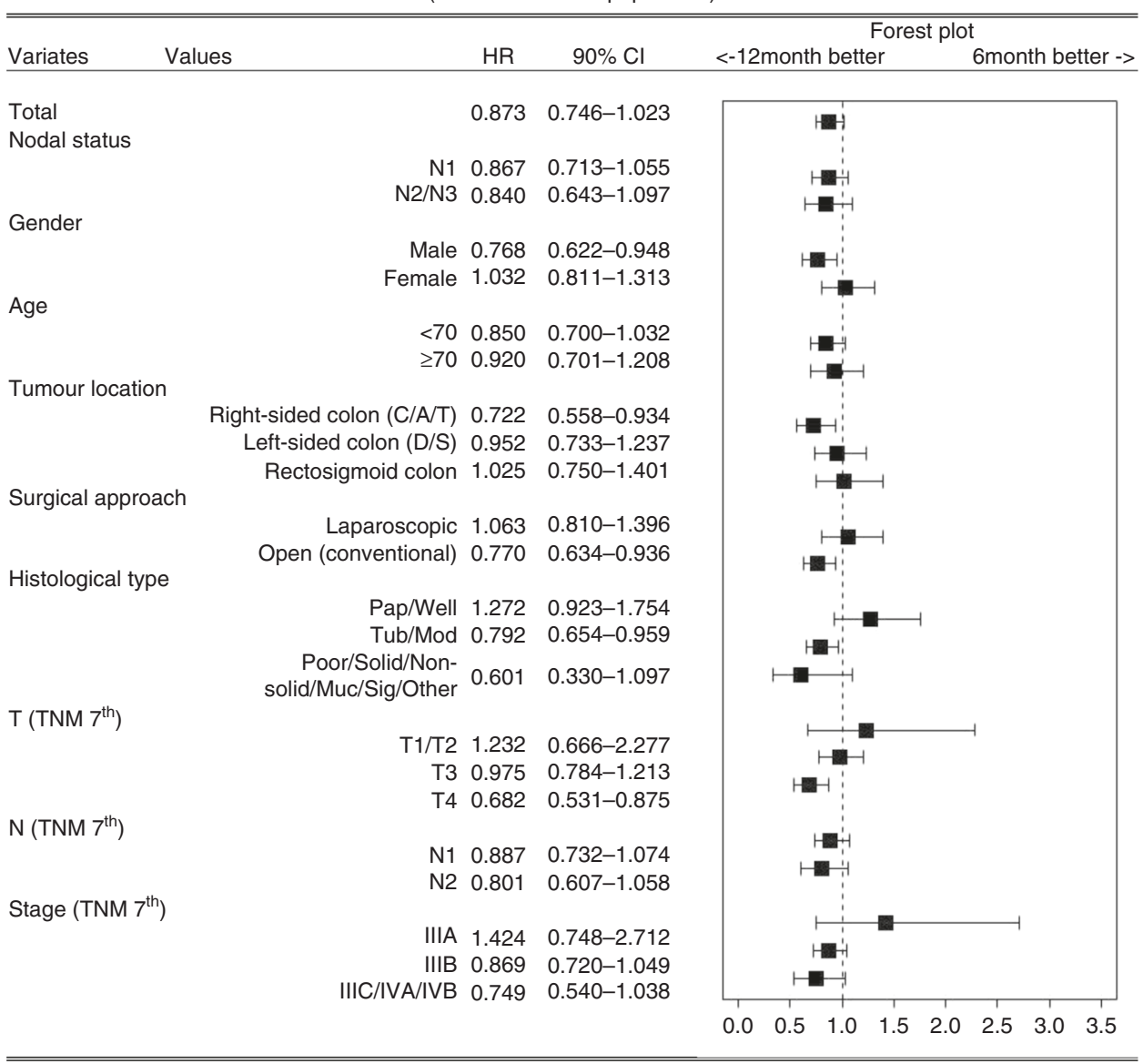

Fig. 4 Subgroup analysis of disease-free survival (intension-to-treat population)

adjuvant chemotherapy for long time and is adopted as one alternative regimen in the latest Japanese guideline. ${ }^{18}$

Recently, the result of IDEA, prospective, pre-planned, global pooled analysis of six randomised phase III trials, comparing the efficacy of 3 and 6 months FOLFOX/CAPOX in postoperative adjuvant chemotherapy for curatively resected stage III colon cancer has been reported. ${ }^{19}$ The non-inferiority of 3 months FOLFOX/Capeox to 6 months was not confirmed in the overall population. However, interestingly, in pre-planned subset analysis in CAPOX group, 3 months of therapy was shown to be as effective as 6 months. The main conclusion obtained from the subgroup analysis of IDEA trial was that 3 months treatment is effective enough for T1-3, N1 low risk stage III patients but not for T4 and/or N2 high risk stage III patients in which longer treatment, 6 months regimen, gave a better survival outcome. Then, we also performed an exploratory analysis on the DFS hazard ratio of $12 \mathrm{M}$ and $6 \mathrm{M}$ group in low-risk and high-risk subpopulations as same as the subgroup analysis performed in IDEA trial. We defined a lowrisk group as patients with $\mathrm{T} 1, \mathrm{~T} 2$, or $\mathrm{T} 3$ and $\mathrm{N} 1$ disease and a highrisk group as patients with T4, N2 or both as same as IDEA. Hazard ratio in low-risk group was 1.007 (95\% Cl: $0.753-1.345, p=0.9650$ ) and there was no difference in DFS between $6 \mathrm{M}$ and $12 \mathrm{M}$ capecitabine administration. However, hazard ratio in high-risk group was 0.746 (95\% Cl: $0.582-0.957, p=0.0208$ ). This result is consistent with the result shown in IDEA trial and suggest that longer treatment may be beneficial in high risk cases.

Then, how should we interpret the result in the present study. Our result suggesting more favourable outcome of longer administration of capecitabine seems to be against the current stream in this area. In regard to this point, our opinion is that we probably should consider the meaning and/or acting mechanism of post-operative adjuvant chemotherapy for CRC using oxaliplatin-containing regimen and oral $5 \mathrm{FU}$ regimen separately. There might be the difference in the site and/or timing of recurrence between shorter administration of FOLFOX/CAPOX and longer administration of capecitabine alone. Further investigation is clearly needed.

Furthermore, Liu et al. ${ }^{20}$ showed that even patients with advanced cancer prefer oral chemotherapy to intravenous chemotherapy with the guarantee of equivalent efficacy. Of course, considering the poor tolerance of $2500 \mathrm{mg} / \mathrm{m}^{2}$ of capecitabine particularly reported in western elderly patients, an appropriate dose modification of capecitabine is very important in practice as described. ${ }^{15}$ In the future, the comparative study of prolonged administration of oral 5-FU monotherapy with 3 or 6 months oxaliplatin-based regimen would be of great interest, although such study is very hard to be conducted realistically.

Another important result drawn from this study is the good prognostic outcome in terms of DFS, RFS, or OS. As regards efficacy, the 5-year OS of the $12 \mathrm{M}$ group in the present study was high as $87.6 \%$, while those in the global studies using oxaliplatinbased chemotherapy were $76.3 \%$ for FOLFOX4 in MOSAIC, ${ }^{11}$ $80.2 \%$ for FLOX in NSABP-C07, ${ }^{12}$ and $77.6 \%$ for XELOX in XELOXA. ${ }^{13}$ There are several possible biases for these results, including the difference in surgical or postoperative pathological procedures between countries. Recently, Shimada et al. ${ }^{21}$ reported 
that the optimal adjuvant chemotherapy should be chosen based on the risk of recurrence in each country, and Tsuji et al. ${ }^{22}$ also reported that the optimal adjuvant chemotherapy for CRC differed between Japanese and western strategies. In addition, the benefit of adjuvant chemotherapy in CRC itself should be re-evaluated in various aspects. ${ }^{23}$ Considering that 12 months capecitabine with better outcomes than 6 months is not associated with long-term neurotoxicity, this prolonged regimen might be considered as one alternative in the adjuvant setting for stage III CRC patients who refuse any additional toxicity of oxaliplatin or are not able to receive it.

In conclusion, the present study failed to show the superiority of 12 months capecitabine to 6 months regimen in terms of DFS. However, OS and RFS was statistically higher in the $12 \mathrm{M}$ group. Thus, the optimal duration of adjuvant chemotherapy, particularly for oral fluoropyrimidine, for stage III CRC needs further investigation. And 12 months of capecitabine monotherapy, along with 3 months of CAPOX with limited neurotoxicity, could be proposed as a treatment option without neurotoxicity. This paper was presented in part at the annual meeting of the European Society of Medical Oncology, Copenhagen, Denmark, October 7-11, 2016.

\section{ACKNOWLEDGEMENTS}

We are grateful to all the patients who participated in the JFMC37-0801 trial and their families, as well as to all of the co-investigators and their staff for their cooperation for this study. The authors also thank Dr. Eiji Oki of Department of Surgery and Science, Graduate School of Medical Sciences, Kyushu University for his valuable comments, and Ms. Yukari Kawamura, Mr. Yuya Ohtake and Mr. Masaru Mutoh of the Japanese Foundation for Multidisciplinary Treatment of Cancer for their substantial contribution on data management and statistical analysis. Medical writing assistance was provided by Editage and was partly funded by Hyogo College of Medicine. This study was supported by Japanese Foundation for Multidisciplinary Treatment of Cancer (JFMC), a non-profit organization for investigator-initiated studies. Part of the study was funded by an unrestricted research grant provided by Chugai Pharmaceutical, Ajinomoto, Astellas Pharma, Bristol-Myers Squibb, Daiichi Sankyo, Eisai, KUREHA, Kyowa Hakko Kirin, Nippon Kayaku, Nippon Shinyaku, ONO PHARMACEUTICAL, Pfizer, Shionogi, Sumitomo Dainippon, TAIHO PHARMACEUTICAL, Takeda Pharmaceutical, TSUMURA, Wyeth, and Yakult Honsha.

\section{AUTHOR CONTRIBUTIONS}

N.T., H.M., S. Sad and J.S. were responsible for conception and design of the study. C. $H$. and T.Y. contributed to the design and the final interpretation of statistical analysis. S. Saj and Y.M. contributed to the funding. N.T., K.M., M.I. and N.B. performed the data analysis. N.T., K.K., A.M., T.S., K.Y., R.N., S.Y. and S. Sad contributed to the patient data collection. All authors contributed to the interpretation of the data, the preparation and revision of the manuscript, and approved the final version.

\section{ADDITIONAL INFORMATION}

Supplementary information is available for this paper at https://doi.org/10.1038/ s41416-019-0410-0.

Competing interests: N.T. received research funding from Taiho Pharmaceutical Co. and Chugai Pharmaceutical Co. T.Y. received honoraria from Takeda, Chugai, Taiho, Boehringer Ingelheim. He also received funding from Takeda, Taiho. He has a consulting/advisory role in Gilead Science, Bayer. K.Y. received honoraria from Taiho, Chugai, Takeda, Lilly, Daiichi Sankyo, Ono, Merck Serono, Johnson\&Johnson, Covidien, Bayer Yakuhin, Eisai, Olympus, Terumo, Bristol Myers Squibb Japan, Otsuka, Sanofi, Denka, Nippon Kayaku, MSD, and Yakult Honsha. He also received research funding from Sanofi, Yakult Honsha, Chugai, Takeda, Lilly, Ono, Taiho, Daiichi Sankyo, Johnson\&Johnson, Covidien, Otsuka, Nippon Kayaku, Tsumura, Asahi Kasei, Eisai, Kyowa Hakko Kirin Astellas, Toyama Chemical, $\mathrm{KCl}$, and Abbot Japan. N.B. received honoraria from Taiho, Chugai, Eli Lilly, Ono, and Bristol Myers Squibb. He also received research funding from Taiho, Ono, and Bristol Myers-Squibb. S.Y. received honoraria and research funding from Chugai and Taiho. H.M. received honoraria and research funding from Chugai. K.M. received honoraria from Chugai, Yakult, Taiho, Takeda, Eli Lilly, and Merck-Serono. He also received research funding from MSD, Daiichi Sankyo, Ono, Shionogi, Kyowa Hakko Kirin, and Gilead Sciences. He has a consulting/advisory role in Ono and Bayer. M.l. received honoraria from Taiho, Chugai, and Merck-Serono and research funding from Taiho and Yakult. He has a consulting/advisory Role in Taiho. J.S. received honoraria from Tsumura and Chugai and has a consulting/advisory role in Takeda. The remaining authors declare no competing interests.

Data availability: All data and materials are available by inquiring to Japanese Foundation for Multidisciplinary Treatment of Cancer (JFMC).

Ethics approval and consent to participate: Each participating institute's institutional review board or ethics committee approved the study. The trial followed the principles of the Declaration of Helsinki and the Good Clinical Practice Guidelines of the International Conference on Harmonisation. All patients provided written informed consent. Each participating institute's institutional review board or ethics committee approved the study (see Supplementary Table 1 for a list of institutes).

Publisher's note: Springer Nature remains neutral with regard to jurisdictional claims in published maps and institutional affiliations.

\section{REFERENCES}

1. Ferlay, J. et al. Cancer incidence and mortality worldwide: Sources, methods and major patterns in GLOBOCAN 2012. Int. J. Cancer 136, E359-E386 (2015).

2. Projected Cancer Statistics, 2017. Cancer Information Service http://ganjoho.jp/ en/public/statistics/short_pred.html Accessed 20 Dec 2017.

3. Gray, R. G. et al. QUASAR Collaborative Group: Comparison of flourouracil with additional levamisole, higher-dose folinic acid, or both, as adjuvant chemotherapy for colorectal cancer: a randomised trial. Lancet 355, 1588-1596 (2000).

4. Haller, D. G. et al. Phase III study of fluorouracil, leucovorin, and levamisole in high-risk stage II and III colon cancer: final report of Intergroup 0089. J. Clin. Oncol. 23, 8671-8678 (2005).

5. André, T. et al. Semimonthly versus monthly regimen of fluorouracil and leucovorin administered for 24 or 36 weeks as adjuvant therapy in stage II and III colon cancer: Results of a randomized trial. J. Clin. Oncol. 21, 2896-2903 (2003).

6. Twelves, C. et al. Capecitabine as adjuvant treatment for Stage III colon cancer. $N$. Engl. J. Med 352, 2696-2704 (2005).

7. Lembersky, B. C. et al. Oral uracil and tegafur plus leucovorin compared with intravenous fluorouracil and leucovorin in stage II and III carcinoma of the colon: results from National Surgical Adjuvant Breast and Bowel Project Protocol C-06. J. Clin. Oncol. 24, 2059-2064 (2006)

8. The National Cancer Institute Physician Data Query (NCI PDQ) www.cancer.gov/ publications/pdq

9. Sakamoto, J. et al. Meta-Analysis Group of the Japanese Society for Cancer of the Colon and Rectum and the Meta-Analysis Group in Cancer: efficacy of oral adjuvant therapy after resection of colorectal cancer: 5 -year results from three randomized trials. J. Clin. Oncol. 22, 484-492 (2004).

10. Hamada, C. et al. Does 1 year adjuvant chemotherapy with oral 5-FUs in colon cancer reduce the peak of recurrence in 1 year and provide long-term OS benefit? Jpn. J. Clin. Oncol. 41, 299-302 (2011).

11. André, T. et al. Multicenter International Study of Oxaliplatin/5-Fluorouracil/Leucovorin in the Adjuvant Treatment of Colon Cancer (MOSAIC) Investigators: oxaliplatin, fluorouracil, and leucovorin as adjuvant treatment for colon cancer. $\mathrm{N}$. Engl. J. Med. 350, 2343-2451 (2004).

12. Kuebler, J. P. et al. Oxaliplatin combined with weekly bolus fluorouracil and leucovorin as surgical adjuvant chemotherapy for stage II and III colon cancer: results from NSABP C-07. J. Clin. Oncol. 25, 2198-2204 (2007).

13. Schmoll, H. J. et al. Capecitabine plus oxaliplatin (XELOX) versus bolus 5-fluorouracil/leucovorin (5-FU/LV) as adjuvant therapy for stage III colon cancer: Survival follow-up of study NO16968 (XELOXA). J. Clin. Oncol. 30, abstr 388 (2012).

14. De Gramont, Ai et al. GERCOR (French Oncology Research Group): from chemotherapy to targeted therapy in adjuvant treatment for stage III colon cancer. Semin. Oncol. 38, 521-532 (2011).

15. Suto, T. et al. Preplanned safety analysis of the JFMC37-0801 trial: a randomized phase III study of six months versus twelve months of capecitabine as adjuvant chemotherapy for stage III colon cancer. Int. J. Clin. Oncol. 22, 494-504 (2017).

16. Japanese Society for Cancer of the Colon and Rectum: Japanese Classification of Colorectal Carcinoma, 7th edn. ( $2^{\text {nd }}$ edn for English version) Kanehara \& Co., Ltd. Tokyo, Japan, 2009.

17. Sadahiro, S. et al. Randomized phase III trial of treatment duration for oral uracil and tegafur plus leucovorin as adjuvant chemotherapy for patients with stage 
Phase III randomised trial comparing 6 vs. 12-month of capecitabine as...

$\mathrm{N}$ Tomita et al.

696

IIB/III colon cancer: final results of JFMC33-0502. Ann. Oncol. 26, 2274-2280 (2015).

18. Watanabe, T. et al. Japanese Society for Cancer of the Colon and Rectum (JSCCR) guidelines 2010 for the treatment of colorectal cancer. Int. J. Clin. Oncol. 17, 1-29 (2012).

19. Grothey, A. et al. Duration of adjuvant chemotherapy for stage III colon cancer. New Engl. J. Med. 378, 1177-1188 (2018).

20. Liu, G., Franssen, E., Fitch, M. I. \& Warner, E. Patients preference for oral versus intravenous palliative chemotherapy. J. Clin. Oncol. 15, 110-115 (1997).

21. Shimada, Y. et al. Randomised phase III trial of adjuvant chemotherapy with oral uracil and tegafur plus leucovorin versus intravenous fluorouracil and levofolinate in patients with stage III colorectal cancer who have undergone Japanese D2/D3 lymph node dissection: final results of JCOG0205. Eur. J. Cancer 50, 2231-2240 (2014).

22. Tsuji, Y. \& Sugihara, K. Adjuvant chemotherapy for colon cancer: the difference between Japanese and western strategies. Expert. Opin. Pharmacother. 17, 783-790 (2016).
23. Pahlman, L. A. et al. Should the benefit of adjuvant chemotherapy in colon cancer be re-evaluated? J. Clin. Oncol. 34, 1297-1301 (2016).

(c) (i) Open Access This article is licensed under a Creative Commons Attribution 4.0 International License, which permits use, sharing, adaptation, distribution and reproduction in any medium or format, as long as you give appropriate credit to the original author(s) and the source, provide a link to the Creative Commons license, and indicate if changes were made. The images or other third party material in this article are included in the article's Creative Commons license, unless indicated otherwise in a credit line to the material. If material is not included in the article's Creative Commons license and your intended use is not permitted by statutory regulation or exceeds the permitted use, you will need to obtain permission directly from the copyright holder. To view a copy of this license, visit http://creativecommons. org/licenses/by/4.0/.

(c) The Author(s) 2019 